Provided for non-commercial research and education use. Not for reproduction, distribution or commercial use.

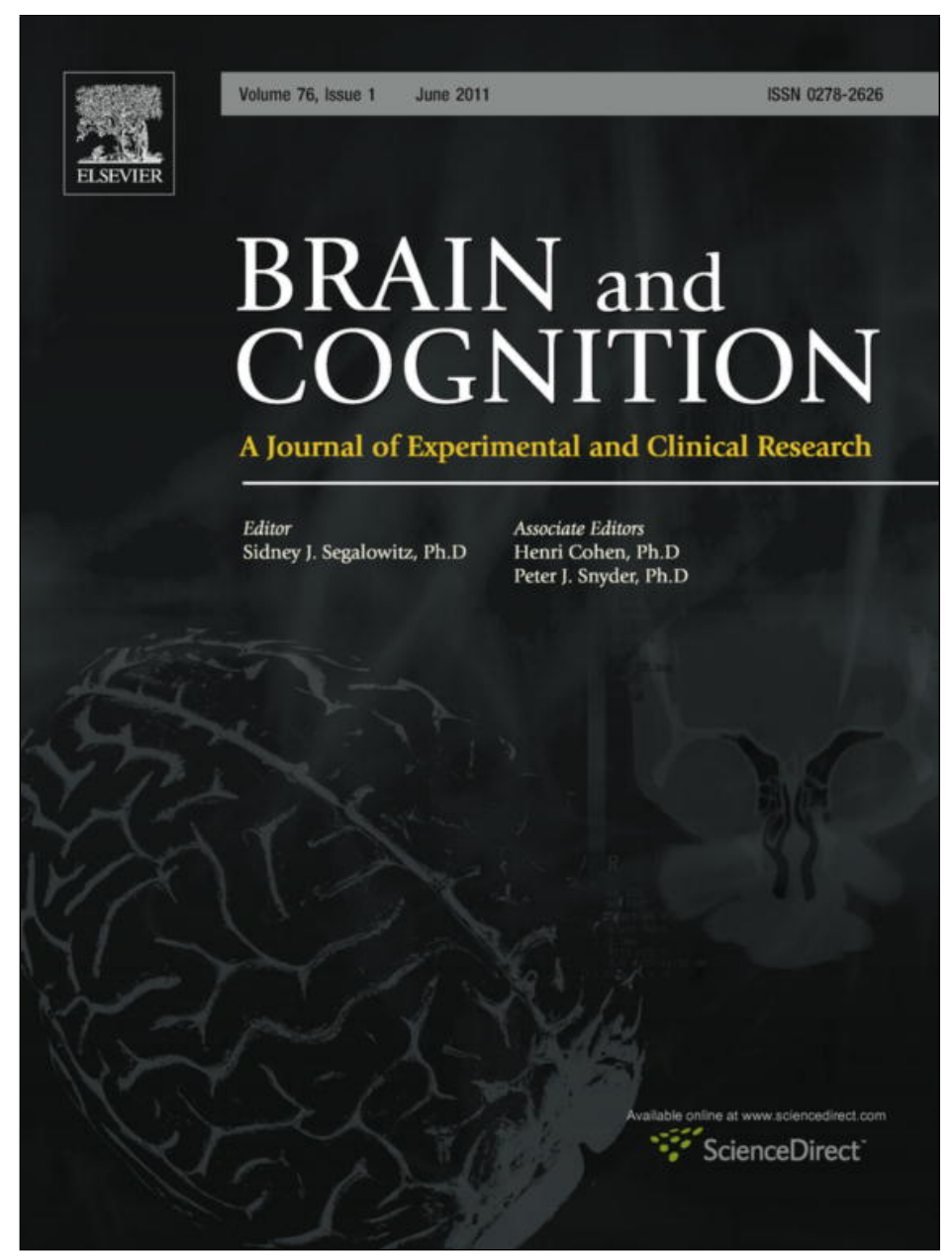

This article appeared in a journal published by Elsevier. The attached copy is furnished to the author for internal non-commercial research and education use, including for instruction at the authors institution and sharing with colleagues.

Other uses, including reproduction and distribution, or selling or licensing copies, or posting to personal, institutional or third party websites are prohibited.

In most cases authors are permitted to post their version of the article (e.g. in Word or Tex form) to their personal website or institutional repository. Authors requiring further information regarding Elsevier's archiving and manuscript policies are encouraged to visit:

http://www.elsevier.com/copyright 


\title{
The influence of contour fragmentation on recognition memory: An event-related potential study
}

\author{
Mathieu B. Brodeur*, J. Bruno Debruille, Louis Renoult, Marie Prévost, Emmanuelle Dionne-Dostie, \\ Lisa Buchy, Martin Lepage
}

Douglas Mental Health University Institute, E Department of Psychiatry, McGill University, Montreal, Canada

\section{A R T I C L E I N F O}

\section{Article history:}

Accepted 21 February 2011

Available online 21 March 2011

\section{Keywords:}

Event-related potentials

Fragmentation

Closure

Episodic memory

Symmetry

Vision

\begin{abstract}
A B S T R A C T
The present study was carried out to examine how the event-related potentials to fragmentation predict recognition success. Stimuli were abstract meaningless figures that were either complete or fragmented to various extents but still recoverable. Stimuli were first encoded as part of a symmetry discrimination task. In a subsequent recognition phase, encoded stimuli were presented complete along with never presented stimuli and participants performed an old/new discrimination task. Fragmentation stimuli elicited more negative ERPs than complete figures over the frontal, central and parietal areas between 180 and $260 \mathrm{~ms}$, and over the occipito-temporal areas between 220 and $340 \mathrm{~ms}$. Only this latter effect was modulated as a function of whether stimuli were recognized or not during the recognition phase of the memory test. More specifically, the effect occurred for stimuli that were later forgotten and was absent for stimuli that were later recognized. This ERP to fragmentation, the occipito-temporal $N_{\text {frag, }}$ possibly reflects the brain response to encoding difficulty, and is thus predictive of recognition performance.
\end{abstract}

(C) 2011 Elsevier Inc. All rights reserved.

\section{Introduction}

In everyday life, people can recognize objects viewed a second time despite first perceiving them in impoverished conditions. This happens because the perception taking place in impoverished conditions is generally extrapolated well beyond the available information captured by the retina. Sometimes, this perception may have precedence over the perception of available information and induce a bias with respect to what will later be remembered. This memory bias was reported by Foley and colleagues (1997). Their participants were first shown complete and fragmented pictures of objects and, later, with names of these objects. When asked to use these words for recollecting the picture version (i.e., complete or fragmented) from memory, participants tended to over-categorized fragmented objects as having been presented complete. In a different approach, Snodgrass and her colleagues used recognition (Snodgrass \& Hirshman, 1994) and implicit fragment completion (Snodgrass \& Feenan, 1990) memory tasks to verify whether fragmented objects could be recollected as well as complete ones. In the former study, subjects encoded line drawings that were complete or fragmented by a partial deletion of their

\footnotetext{
* Corresponding author. Address: Brain Imaging Group, Douglas Mental Health University Institute, FBC Pavilion, 6875 Boul, LaSalle, Verdun, Québec, Canada H4H 1R3. Fax: +1 5148884064 .

E-mail addresses: mathieu.brodeur@gmail.com, mathieu.brodeur@douglas. mcgill.ca (M.B. Brodeur).
}

contours. Depending on the magnitude of the deletion, imagining the stimulus as complete and identifying it was either possible (i.e., recoverable stimuli) or not (i.e., unrecoverable stimuli). In the recognition phase, participants were presented with the complete version of the encoded objects and with never seen (i.e., new) objects. Recoverable stimuli were better recognized than unrecoverable stimuli, suggesting that cognitive processes in response to fragmentation, which likely contributed to the integration of the fragments into a well defined global form, may improve recognition.

The contribution of fragmentation processing on recognition can be directly assessed by computing the event-related potentials (ERPs) evoked across different levels of fragmentation and by examining whether the resulting ERP effects predict recognition performance. Fragmentation processing has been associated with several ERP components that all had negative polarity and peaked between 200 and $450 \mathrm{~ms}$. The $\mathrm{Ncl}$ (standing for negativity to closure) is one of these ERPs. The first Ncl to have been described started approximately at $230 \mathrm{~ms}$ and peaked around $290 \mathrm{~ms}$ over occipito-temporal scalp sites (Doniger et al., 2000). It was obtained by presenting the stimuli following the ascending method of limits (Snodgrass, Smith, Feenan, \& Corwin, 1987). Stimuli first appear in a very fragmented version and, gradually, several contour fragments are added until participants succeed at object identification. Doniger et al. (2000) found that the voltage of the Ncl became incrementally more negative as levels of fragmentation approached the level of identification, with a particularly marked 
change of amplitude at identification. A second well-established ERP in response to fragmentation is the N350, a frontal negativity first described by Schendan and Kutas (2002). In one task, pictures were presented according to the ascending method of limits. Pictures presented two fragmentation levels prior to identification elicited a larger N350 than identified pictures, a result opposite to the Ncl modulation, which was rather larger at identification. In other tasks, an N350 modulation was also found between unidentified and identified objects that were fragmented at the same level and which were therefore controlled for physical dissimilarities. Schendan and Kutas (2002) assumed that their frontal $\mathrm{N} 350$ and the temporo-occipital $\mathrm{Ncl}$ reflect identical processes and argued that their difference of scalp distribution was due to the use of different electrodes of reference (on the nose in Doniger et al., 2000 and on the left mastoid, recomputed offline to the averaged mastoids in Schendan \& Kutas, 2002). Negativities revealed with comparable paradigms do not systematically peak at the same latencies. For instance, the Ncl recorded by Sehatpour and colleagues (2006) peaked at $320 \mathrm{~ms}, 30 \mathrm{~ms}$ later than the first reported $\mathrm{Ncl}$. Latencies might thus be highly sensitive to subtle variables across experiments and to differences between groups of participants. Differences between the Ncl and the N350, however, go beyond simple difference of latencies and it would be premature to conclude that they are the expression of the same brain processes (see also Sehatpour et al., 2006 for further arguments).

In the ERP studies described above, the landmark signaling when the brain has successfully managed fragmentation is the accurate identification of the stimulus or its recognition from a set of stimuli previously encoded. One may assume that prior to the identification, knowledge associated with the stimuli is well controlled and causes no confounding effect on fragmentation processing. In fact, the inability to identify a stimulus does not signify that there is no associated knowledge. It is indeed possible that, prior to identification, some pieces of the contour are locally integrated into a meaningful but partial structure which alone is insufficient to allow full identification of the object (Doniger et al., 2000). For instance, perceiving the legs of a fragmented horse is enough to determine that the stimulus is an animal but not sufficient to identify the horse. It is also possible that the perception of only several fragments is sufficient to correctly identify the whole object. Therefore, identification does not necessarily mean that fragments have all been fully processed or closed.

The potential effect of associated knowledge on the identification of the stimuli can be attenuated by using abstract figures instead of recognizable objects. Fragments of gabors (cosine patches within a gaussian window) (see Hess \& Field, 1999 for a review) delineating very simple shapes elicit negative components of the ERPs but they generally peak earlier than the negativities obtained with recognizable objects (Mathes, Trenner, \& Fahle, 2006). A second way to control for potential effects of associated knowledge consists in modulating fragmentation within the limits of recoverability. In such conditions, all stimuli benefit from the contribution of associated knowledge equally and only fragmentation varies across conditions. ERP modulations across recoverable stimuli have not been extensively examined. Stuss and colleagues (1992) compared the ERPs to stimuli that were incomplete to different extents but were all correctly identified. Their results indicate that between 250 and $450 \mathrm{~ms}$, at $\mathrm{Cz}$, the amplitude was more negative to stimuli that were the most incomplete. Taking a different approach, Viggiano and Kutas (1998), Viggiano and Kutas (2000) compared the ERPs evoked at the identification level with those at one higher level with more fragments added to the stimulus. Consistent with the results of Stuss and colleagues (Stuss et al., 1992), the amplitude was more negative for the identification level, thus for the most fragmented level. The authors mentioned that this effect was restricted to posterior sites, but the electrodes, as well as the latencies, were not specified more precisely. A visual inspection of the ERPs depicted in their Fig. 8 (p. 114) (Viggiano \& Kutas, 2000), however, suggests that the effect peaked somewhere between 200 and $300 \mathrm{~ms}$.

The present study tested whether the ERP effect to fragmentation are predictive of the subsequent recognition in a memory recognition task. Figures were abstract and meaningless and the encoding task targeted a physical aspect of the stimuli, namely their symmetry (Boucart \& Humphreys, 1994). To further control for semantic effect, fragmentation was manipulated within the limits of recoverability. Figures could thus all be imagined complete but the effort to achieve this changed as a function of fragmentation levels. Fragmentation elicited a brain correlate referred to as the $N_{\text {frag }}$ (standing for negativity to fragmentation). The magnitude of the $N_{\text {frag }}$ can only be assessed if contrasted to the ERPs evoked by a control figure involving no fragmentation processing. This latter was a complete figure, and the comparison of its ERPs with the $N_{\text {frag }}$ was referred to as the $N_{\text {frag }}$ effect.

Based on the results of Stuss and colleagues (1992) and Schendan and Kutas (2002), we expected an $N_{\text {frag }}$ effect to be elicited mainly over central and posterior scalp areas. The effect of fragmentation on encoding was tested by comparing the $N_{\text {frag }}$ effect of stimuli subsequently recognized with the $N_{\text {frag }}$ effect of stimuli subsequently forgotten. Predictions about these effects depend on the nature of the processes underlying the $N_{\text {frag }}$ and how efficient they are. On the one hand, the $N_{\text {frag }}$ may reflect a successful perceptual closure of the figure that necessarily improves recognition. Accordingly, the $N_{\text {frag }}$ effect should be greater between the complete and fragmented stimuli that were subsequently recognized. On the other hand, the $N_{\text {frag }}$ could reflect a brain response to a perceptual problem and the compensatory processes engaged to overcome this problem. Since this problem would likely hinder subsequent recognition, the $N_{\text {frag }}$ effect should be greater for figures that were subsequently forgotten.

\section{Methods}

\subsection{Subjects}

Twenty-five participants ( 9 females) aged between 21 and 30 were recruited, though one had to be excluded from the analyses because of his performance (see the behavioral analyses section). All met the inclusion criteria typically used for ERP studies, in that they were right-handed and had normal or corrected-to-normal vision. All participants reported that they and their first-degree relatives had no history of neurological or psychiatric disorders. Participants provided written consent on a form approved by the Douglas Institute Research Ethics Board.

\subsection{Stimul}

Stimuli were 400 abstract figures not associated with a priori knowledge. Figures were built from an $8 \times 8$ grid measuring $6 \mathrm{~cm} \times 6 \mathrm{~cm}$ on the computer screen $\left(8.5^{\circ}\right.$ of visual angle) (see Brodeur, Pelletier, \& Lepage, 2008b for further details). Example stimuli are depicted in Fig. 1. Half of the figures were symmetric across the vertical axis and half were asymmetric. The contours of 150 figures were fragmented by means of creating gaps with lengths totaling $70 \%$ of the remaining fragment lengths. None of these fragments were removed in 50 figures, the Frag-0\% figures (e.g., the first figure of the encoding session in Fig. 1). In 50 other figures, $15 \%$ of the fragments were removed (e.g., the third figure of the encoding session in Fig. 1) and in 50 different figures 25\% were removed (e.g., the fourth figure of the encoding session in Fig. 1). These figures constitute what we have labeled the 
Frag-15\% and Frag-25\% conditions, respectively. The proportion of removed fragments is far from the proportion necessary to prevent identification of the Snodgrass and colleagues (1987) stimuli. Thus, all fragmented stimuli were recoverable including those in the most fragmented condition. The meaningfulness of the figures was verified by presenting all figures to 195 undergraduate students and asking them to note those that evoked something meaningful. An evocative score was computed for each figure by averaging the number of students for whom the figure was meaningful and converting this value into a percentage. Evocative scores were $14 \%$ with the complete (SD: 11 ) and Frag-0\% (SD: 12) figures, and 13\% with the Frag-15\% (SD: 10) and Frag-25\% (SD: 13) figures. No difference between figures reached significance.

\subsection{Procedure}

The experiment was divided in 10 blocks. Blocks each included an encoding and a recognition phase. Fig. 1 illustrates the experimental design. In each encoding phase five complete figures and five figures of each level of fragmentation were randomly presented twice. Duration of the presentation was fixed at $4 \mathrm{~s}$ and was followed by a blank screen with a fixation cross for $1.5 \mathrm{~s}$. Participants were instructed to judge whether figures were symmetric or asymmetric by pressing on two different keys of the keyboard with their right index finger. Symmetry was counterbalanced across figures. Each encoding phase was immediately followed by a recognition task in which the encoded figures as well as 20 novel figures were shown, the latter of which were also counterbalanced for symmetry. All figures were presented in their unfragmented version in the recognition phase. Stimuli were presented for $2.5 \mathrm{~s}$ with an inter-stimulus interval of $3 \mathrm{~s}$. Participants performed an old/new discrimination task and again provided their responses by pressing on two different keys on the keyboard with their right index finger. Total experimental duration was $1 \mathrm{~h}$ and $40 \mathrm{~min}$.

\subsection{EEG acquisition}

The EEG was captured by 28 tin electrodes mounted in an elastic cap of 32 electrodes placed according to the $10-20$ system. Electrodes were also placed on the external canthi and below the eyes to record the EOG and monitor eye blinks and ocular movements. Impedances in all channels were set below $5 \mathrm{k} \Omega$. The signal was referenced to the right ear lobe and re-referenced offline to linked ears. High and low pass filters with half-amplitude cut-offs set at 0.01 and $100 \mathrm{~Hz}$, respectively, were used in addition to a $60 \mathrm{~Hz}$ electronic notch filter. Signals were amplified 20,000 times and digitized at a sampling rate of $500 \mathrm{~Hz}$.

\subsection{Behavioral analyses}

Analyses were conducted on reaction times and percentages of stimuli correctly classified as symmetric/asymmetric in the encoding task and old/new in the recognition task. The Hit rate was the percentage of old figures correctly responded to in the recognition task, and included more symmetric figures than asymmetric figures but the proportion was comparable across conditions (complete: $40 \%$ asymmetric/60\% symmetric, Frag-0\%: 40/60\%, Frag-15\%: 41/59\%, Frag-25\%: 37/63\%). Two-way within-subjects analyses of variance (ANOVAs) were performed, with stimulus fragmentation (4 levels: complete, Frag-0\%, Frag-15\%, Frag-25\%) and subsequent recognition (2 levels: recognized, forgotten) as factors. The symmetry factor was not included in the model as it is not in the focus of our study and would considerably reduce the number of stimuli included in the averaged conditions of the ERP analyses. One participant performed at the chance level on the recognition task.

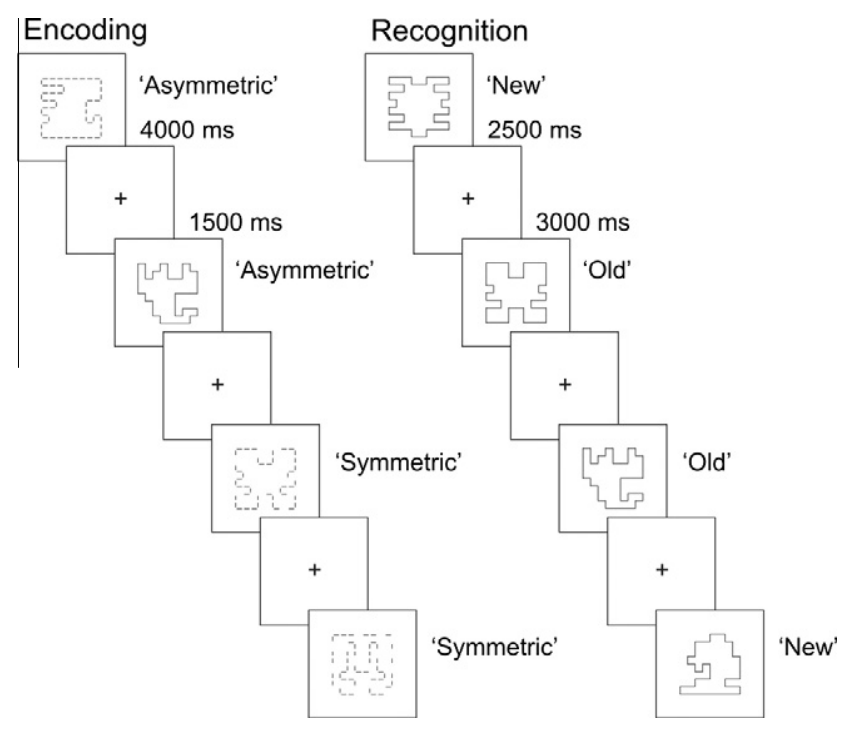

Fig. 1. Experimental design with example stimuli for each condition. Stimuli in the encoding phase were symmetric or asymmetric and were complete or fragmented at various levels. The figures depicted as examples are a Frag-0\% asymmetric figure, a complete asymmetric figure, a Frag-15\% symmetric figure, and a Frag-25\% symmetric figure. All encoded stimuli were presented complete in the recognition phase along with novel stimuli.

He recognized between $50 \%$ and $52 \%$ of the old figures across the four levels of fragmentation and correctly rejected $48 \%$ of the new figures. This participant was thus excluded from the behavioral and ERP analyses.

\subsection{ERP analyses}

Epochs from -200 to 500 ms were extracted from the EEG and submitted to an offline automatic rejection procedure. Rejection criteria were saturations for more than $100 \mathrm{~ms}$ and amplitudes reaching $\pm 100 \mu \mathrm{V}$. No eye artefact correction was used. For each subject, the accepted trials of all blocks were averaged in accordance with the condition to which they belonged. The first and repeated presentation of the stimuli during encoding were averaged together because repetition had no impact $(F(1,24)=0.02$, $p=.8872$ ) within the time-window defined below and because it did not interact with the fragmentation condition $(F(15,360)=$ $1.07, p=.3620$ ). The trials were averaged together as a function of their fragmentation but also as a function of their subsequent recognition. This latter variable was necessary to fulfill our main objective and see whether the brain response to fragmentation during stimulus encoding was determinant for the stimulus at subsequent recognition.

The negativity to fragmentation was identified as the ERP component that was more negative for the fragmentation conditions as compared to the complete condition between 180 and $450 \mathrm{~ms}$. Because our stimuli were visually simpler, this time-window started slightly earlier than those used by Stuss and colleagues (1992) and by Viggiano and Kutas (1998), Viggiano and Kutas (2000). The analyses were conducted on three subsets of electrodes, each of which covered an area where modulation to fragmentation has been reported in the literature. The first subset covered the occipito-temporal area and included the electrodes 02, 01, T6, and T5 (Doniger et al., 2000). The second subset covered the centro-parietal area and included the electrodes Cz, C4, C3, Cp4, Cp3, Pz, P4, P3 (Stuss et al., 1992). The third subset covered the frontal area and included the electrodes Fp2, Fp1, F4, and F3 (Viggiano \& Kutas, 1998; Viggiano \& Kutas, 2000). The negativity found in each of these three subsets had different latencies. Accordingly, different 
time-windows were used for analyzing the modulation to fragmentation. The time-windows were $220-340 \mathrm{~ms}$ for the occipito-temporal subset and $180-260 \mathrm{~ms}$ for the frontal and centro-parietal subset.

The ANOVA model used for each measure (frontal $N_{\text {frag }}$, centroparietal $N_{\text {frag }}$, occipito-temporal $N_{\text {frag }}$ ) had electrode (4 levels in frontal and occipito-temporal subset, and 6 levels in the centroparietal subset), hemiscalp (2 levels: left, right), fragmentation (4 levels: complete, Frag-0\%, Frag-15\%, Frag-25\%), and subsequent recognition (2 levels: recognized, forgotten) as within-subjects factors. The electrodes $\mathrm{Pz}$ and $\mathrm{Cz}$ could not be included in this model because of the hemiscalp factor. A separate analysis was thus conducted with two levels for the electrode factor and no hemiscalp factor. ERP modulation to fragmentation, the $N_{\text {frag }}$ effect, was first visually inspected to delineate the time-windows described above. The $N_{\text {frag }}$ effect was thereafter examined as a function of the subsequent recognition. Additional analyses were also carried out separately for each level of fragmentation to determine the source of the interaction. The Geisser and Greenhouse procedure for violation of sphericity was used to correct degrees of freedom for factors that had more than two levels. The epsilon values, as well as the original degrees of freedom and corrected $p$ values are presented.

\section{Results}

\subsection{Behavioral results}

Behavioral results are illustrated in Fig. 2. Results from all figures irrespective of their subsequent recognition show that 97.0\% (SD: 3.9) of encoding figures were correctly classified as symmetric or asymmetric, demonstrating that subjects had little difficulty performing this task. The scores were $98.2 \%$ (SD: 3.7) for the complete condition, 97.9\% (SD: 3.1) for the Frag-0\% condition, 96.7\% (SD: 4.1) for the Frag-15\% condition, and 95.4\% (SD: 4.6) for the Frag$25 \%$ condition. The high levels of performance substantially reduced the variances and brought the main effect of fragmentation to a statistical significant level $(F(3,69)=7.59, p=.0006)$. The effect of fragmentation did not interact with the subsequent recognition factor $(F(3,69)=0.21)$, and the subsequent recognition effect was not significant $(F(1,23)=0.60)$. When considering reaction time, a main effect of fragmentation was observed, $(F(3,69)=12.63, p<.0001)$. Shortest response times were recorded for complete figures (1259, SD: $470 \mathrm{~ms}$ ) and Frag-0\% figures (1255, SD: $463 \mathrm{~ms}$ ), with a significant increase for Frag-15\% figures (1338, SD: $523 \mathrm{~ms}$ ) as compared to the Frag- $0 \%(F(1,23)=11.81, p=.0023)$ and a significant increase for Frag-25\% figures (1392, SD: $528 \mathrm{~ms}$ ) as compared to the Frag-15\% $(F(1,22)=5.96, p=.0227)$. As with accuracy, there was no main effect of subsequent recognition or interaction between this factor and fragmentation.

In the recognition task, $65.2 \%$ (SD: 12.5 ) of old figures and $69.2 \%$ (SD: 12.7) of new figures were correctly classified. Hit rate was 73.3\% (SD: 14.1) for the complete condition, 70.5\% (SD: 11.3 ) for the Frag-0\% condition, 62.1\% (SD: 10.7) for the Frag-15\% condition, and $54.8 \%$ (SD: 13.8 ) for the Frag-25\% condition. Note that figures of the Frag- $0 \%,-15 \%$, and $-25 \%$ conditions were presented in their complete version in the recognition phase. The main effect of fragmentation was significant $(F(3,69)=24.60, p<.0001)$. $T$-tests indicated that the decrease of accuracy between the complete and the Frag-0\% conditions was not significant $(t(23)=1.46, p=.1589)$, in contrast with the decreases between the Frag-0\% and Frag-15\% $(t(23)=4.91, p=.0001)$, and between Frag-15\% and Frag-25\% $(t(23)=2.68, p=.0134)$, which were both significant. When considering reaction times, a significant main effect of fragmentation was observed $(F(1,22)=18.85, p<.0001)$. Reaction times for recognizing complete (1343, SD: $378 \mathrm{~ms}$ ) and Frag-0\% figures
(1334, SD: $308 \mathrm{~ms}$ ) at encoding did not significantly differ. Figures of the Frag-15\% condition were recognized more slowly (1492, SD: $397 \mathrm{~ms}$ ) than those of the Frag-0\% condition $(t(23)=5.01$, $p<.0001)$ and not significantly faster than the figures of the Frag-25\% condition (1503, SD: $342 \mathrm{~ms})(t(23)=0.35, p=.7323)$.

\subsection{ERPs as a function of fragmentation}

Fig. 3 depicts ERPs to the four levels of fragmentation. The centroparietal and frontal $N_{\text {frag }}$ are perceptible over most of the electrodes. Over the central electrodes, and particularly C4, these effects have the appearance of a negative 'bump' embedded in the slope of the broad P3. The ANOVA conducted in the 180-260 time-window revealed a fragmentation effect at the centro-parietal $(F(3,69)=$ $6.48, p=.0020)$ and frontal subsets $(F(3,69)=7.91, p=.0005)$. The effect was also significant at $\mathrm{Pz}$ and $\mathrm{Cz}(F(3,69)=4.14, p=.0155)$ but not at the occipito-temporal subset. There was no main effect of subsequent recognition (all $F(1,23)<2.81$ ) and this factor did not interact with fragmentation (all $F(3,69)<1.59$ ). The $N_{\text {frag }}$ recorded over occipito-temporal areas started later than the frontal and central $N_{\text {frag. }}$. The occipito-temporal $N_{\text {frag }}$ is the negative deflection embedded in the P3. It is clearly perceptible over the occipital electrodes where the difference of amplitude between the complete condition and the fragmented condition is the greatest in the timewindow of 220-340 ms. Result from the ANOVA conducted for this time-window indicated that the fragmentation effect was significant $(F(3,69)=4.45, p=.0254)$, but that it did not interact with the electrodes and/or the hemiscalp factors. ERP modulations as a function of fragmentation within the $220-340 \mathrm{~ms}$ time-window were also tested with the other subsets of electrodes. No main effect of fragmentation reached significance but the fragmentation and electrode factors interacted significantly at the centro-parietal subset $(F(3,69)=3.63, p=.0170, \varepsilon=.501)$. Further analyses indicated that this interaction was due to a main effect of fragmentation that was found over P4-P3 $(F(3,69)=5.54, p=.0039)$ but not over the other electrodes of the subset.

\subsection{ERPs as a function of recognition}

Fig. 4 depicts the occipito-temporal $N_{\text {frag }}$ as a function of subsequent recognition. It can be seen that the magnitude of the occipitotemporal $N_{\text {frag }}$ effect is larger with figures that are subsequently forgotten than with figures that are subsequently recognized. Result from the ANOVA conducted for the 220-340 ms time-window indicated that the fragmentation $\times$ subsequent recognition interaction was significant $(F(3,69)=4.50, p=.0121, \varepsilon=.768)$. The same ANOVA also reached significance $(F(3,69)=3.49, \quad p=.0307$, $\varepsilon=.707$ ) when conducted on the ERPs to the figures at their first presentation, thus excluding the repeated trials from the grand averages. No such interaction was found at other subsets or in the other time-windows. The fragmentation $\times$ subsequent recognition interactions conducted between the complete and the fragmented conditions were significant between the complete and Frag- $15 \%$ conditions $(F(1,23)=5.51, p=.0278)$ and between the complete and Frag-25\% conditions $(F(1,23)=12.60, p=.0016)$ but not between the complete and Frag- $0 \%$ conditions. Additional analyses were conducted to test separately the fragmentation effect in the recognized and forgotten figures. Results showed that the fragmentation effect was significant with the forgotten figures $(F(3,69)=4.95, p=.0050)$ but not with the recognized ones.

The occipito-temporal $N_{\text {frag }}$ modulation of each level of fragmentation was also statistically tested as a function of subsequent recognition. Significance differences emerged with the complete and the Frag-25\% figures. The former had larger amplitude when subsequently recognized than subsequently forgotten $(F(1,23)=$ $4.43, p=.0473)$. Frag-25\% figures subsequently forgotten evoked 

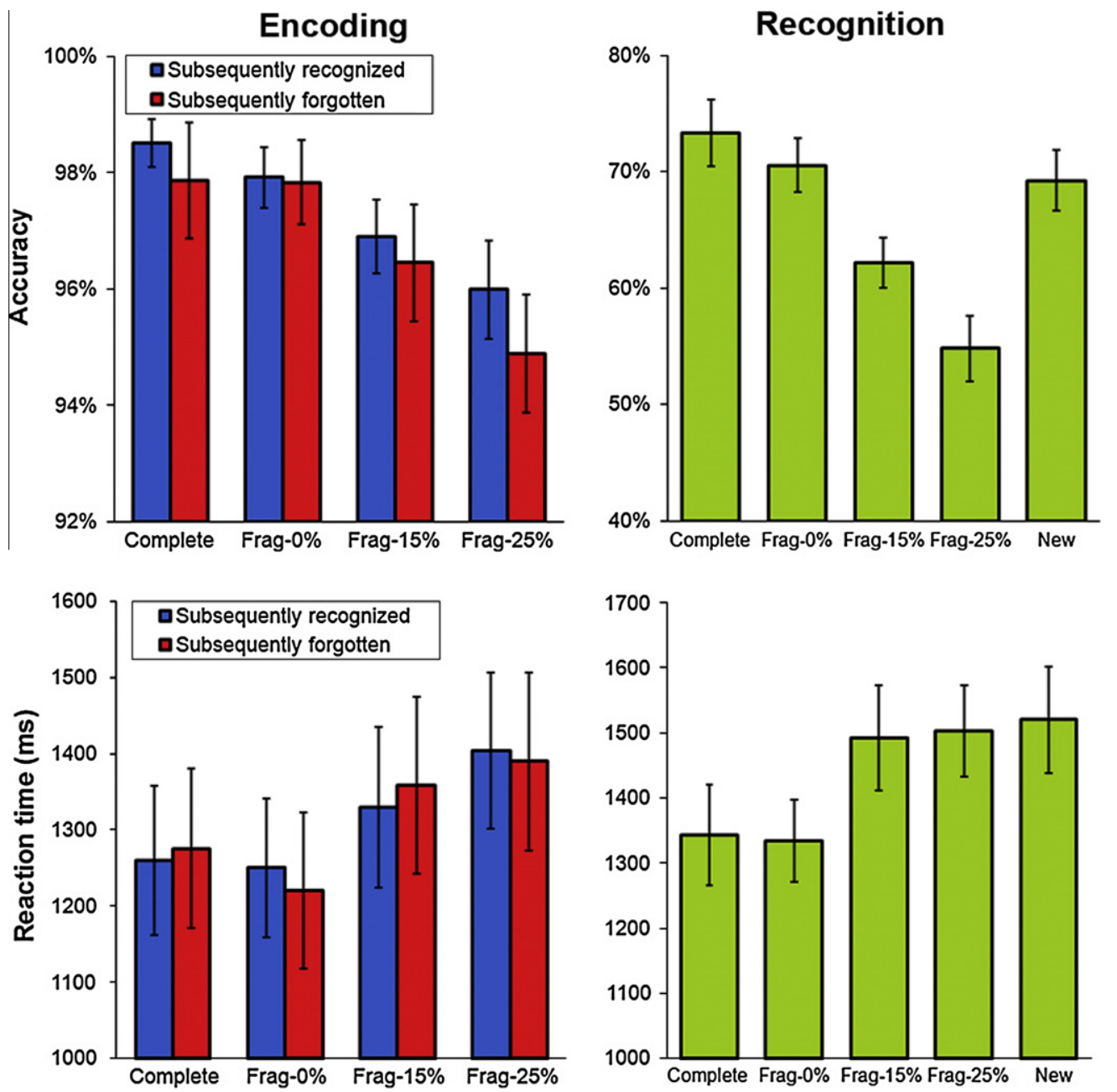

Fig. 2. Behavioral performances. Percentages of encoded figures correctly classified as symmetric and asymmetric as well as reaction times are presented in the left panel as a function of subsequent recognition. Hit rates and reaction times to the recognition task are presented in the right panel. Note that all figures were presented complete during recognition. Conditions refer to the level of fragmentation of figures when presented during encoding.

larger $N_{\text {frag. }}(F(1,23)=6.10, p=.0215)$. Effect of subsequent recognition is presented in Fig. 5 for each fragmentation condition.

\section{Discussion}

Consistent with previous reports (Schendan \& Kutas, 2002; Stuss et al., 1992), the present results showed that the fragmented figures evoked more negative ERPs than complete figures between 180 and 340 ms. This ERP, the $N_{\text {frag, }}$ was not significantly different across fragmented stimuli, thus suggesting that once stimuli are recoverable, the $N_{\text {frag }}$ is no more affected by fragmentation. Further analyses showed that the occipito-temporal $N_{\text {frag }}$ effect starting around $220 \mathrm{~ms}$ varied as a function of subsequent recognition. It was significant for stimuli subsequently forgotten but not significant for stimuli subsequently recognized. This interaction was driven by the complete and Frag-25\% figures which had their $N_{\text {frag }}$ respectively increased and decreased by subsequent forgetting. These results thus suggest that when an occipito-temporal $N_{\text {frag }}$ effect occurred, the chances of recognizing the stimuli were compromised. Several propositions will be discussed below to account for the $N_{\text {frag }}$ effect on the basis of these observations.

\subsection{The effect of recoverability on fragmentation processing}

Symmetry of figures was accurately determined (between 95\% and $98 \%$ ), confirming that fragmented figures were recoverable.
Recoverability, implemented to control for semantic confound effects, had important influences on the results and should be considered in the interpretation of the data. First, it might explain the lack of ERP differences across the three levels of fragmentation. A mental effort was necessary to monitor the fragmentation, as indicated by the occipito-temporal $N_{\text {frag }}$ but this effort was probably kept at a minimum throughout all levels because figures were all recoverable. Greater disparity between the levels of fragmentation might have been needed to observe a change in the occipitotemporal $N_{\text {frag }}$ across the levels of fragmentation. The recoverability of the figures might also account for an important discrepancy between the present results, showing that fragmentation hindered recognition, and those of other studies indicating that at a particular level, fragmentation improves recognition. For instance, Snodgrass and Feenan (1990) showed that complete objects were better primed by moderately fragmented objects than very fragmented and complete objects. This priming effect, which can be considered a preliminary phase to the access of the memory trace (Voss, Baym, \& Paller, 2008), was specifically perceptual as it occurred between physically matched objects but not between primes and targets sharing only conceptual attributes (e.g., pictures vs. words) (Snodgrass \& Kinjo, 1998). According to Kinjo and Snodgrass (2000), moderately fragmented stimuli create a stronger priming effect than complete stimuli because they benefit from an active mode of perception where subjects complete mentally the stimuli. In the present study, subjects likely achieved such 

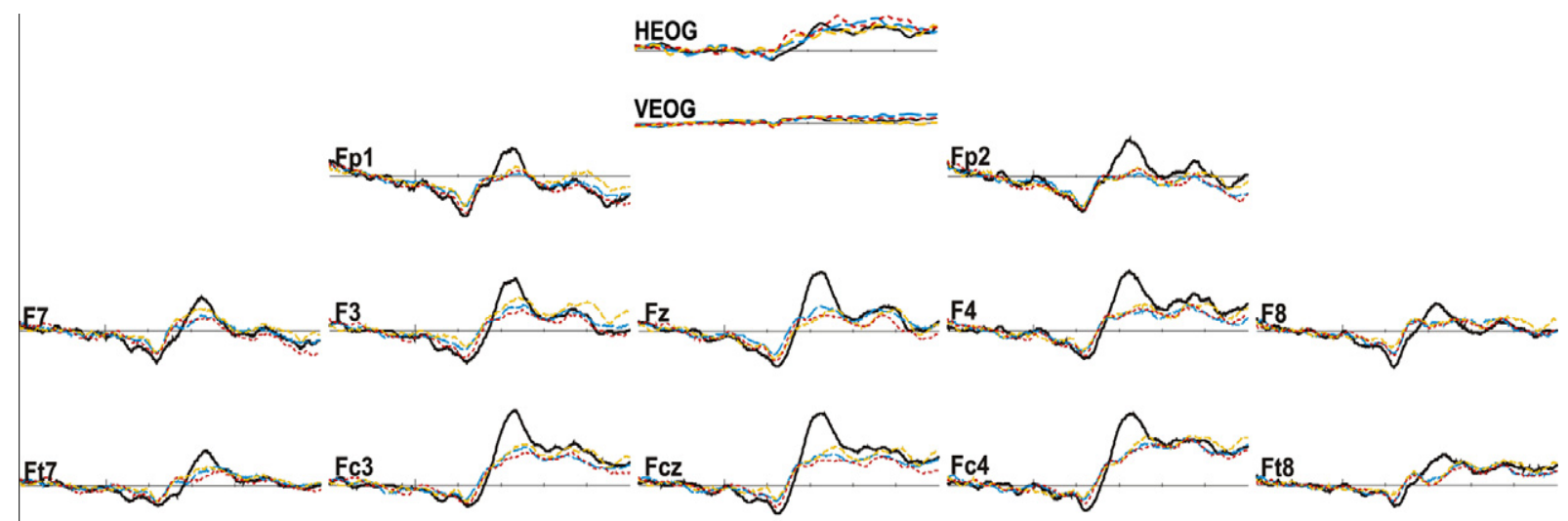

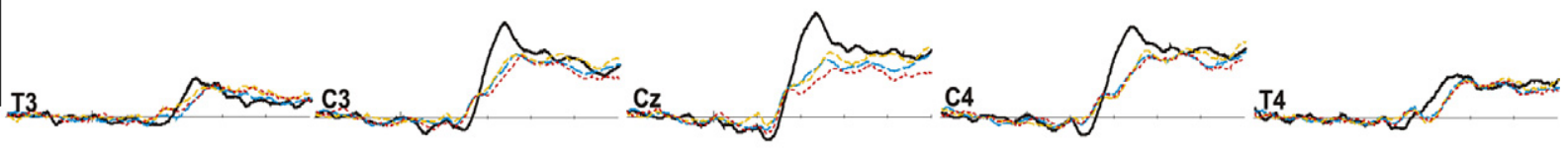

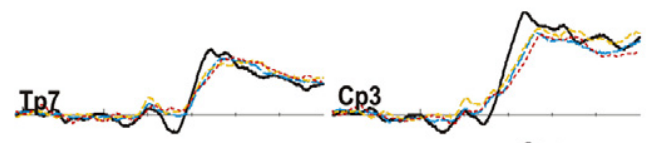

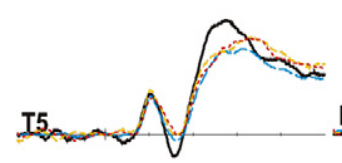

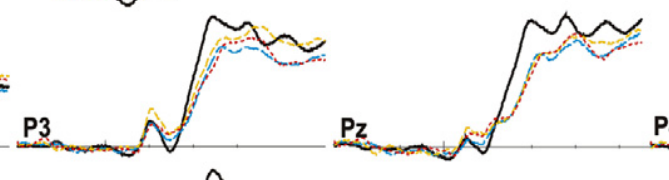<smiles>CCCCCCCCCCCCO</smiles>

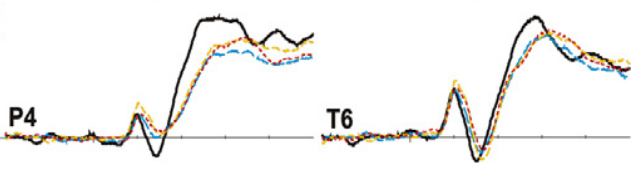

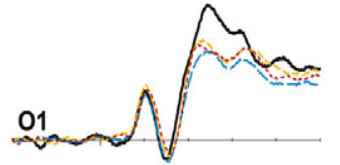

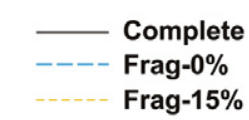

Frag-25\%
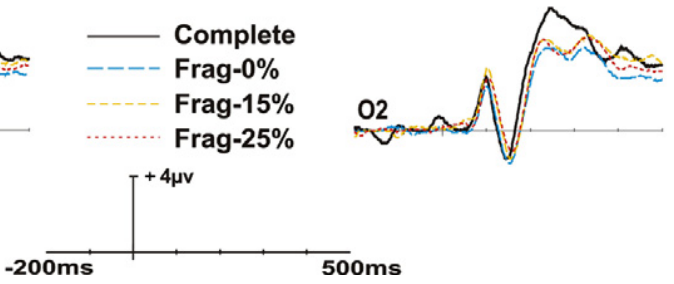

Fig. 3. Grand averaged ERPs $(n=24)$ evoked by the four conditions of fragmentation.

Subsequently recognized
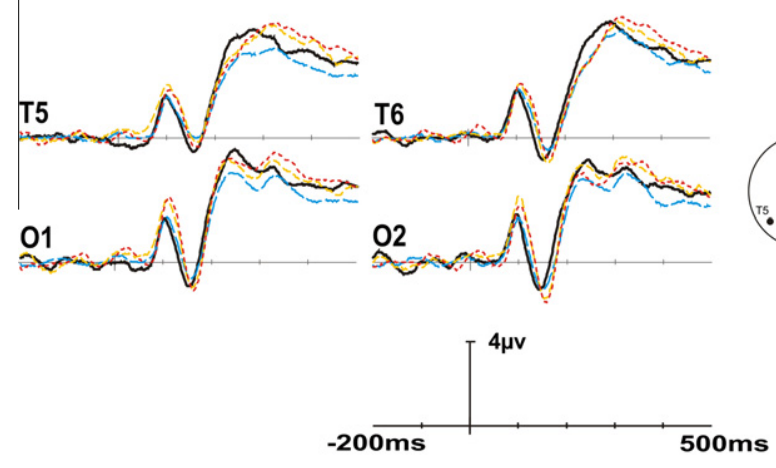

Subsequently forgotten

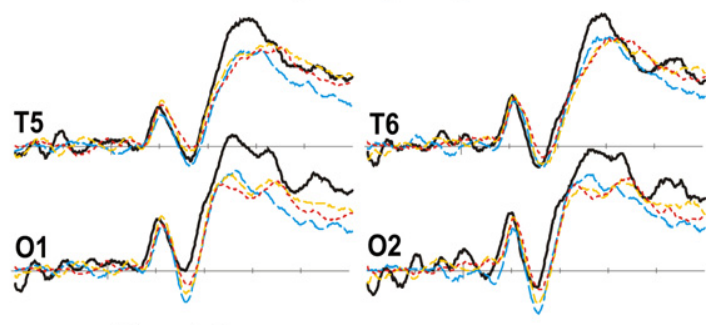

Complete

Frag- $0 \%$

Frag- $15 \%$

Frag- $25 \%$

Fig. 4. Grand averaged ERPs $(n=24)$ evoked by the fragmentation levels as a function of subsequent recognition.

effort since they had to imagine the figures in their complete version while performing the symmetry task during encoding. This effort was probably the same across all levels of fragmentation and was probably insufficiently activated, given the low level of fragmentation, to improve subsequent recognition.

One may have noticed that the behavioral performances and the occipito-temporal $N_{\text {frag }}$ were not modulated similarly across the conditions of fragmentation. Accuracy and reaction times were indeed modulated as a function of fragmentation in contrast with the occipito-temporal $N_{\text {frag, }}$ which only differed between complete and fragmented conditions. The long duration of stimulus presentation might be responsible for the discrepancy between the behavioral and ERP effect as it encouraged more extended cognitive processing that relates to behavior but was not captured by the ERP response within the $N_{\text {frag }}$ time-window. As for the reaction times, they were statistically indistinguishable between the complete and Frag- $0 \%$ conditions, thus suggesting that discriminating symmetric from asymmetric figures was as easy with complete figures as with slightly fragmented figures. A similar conclusion was proposed by Boucart and colleagues (1994) (Boucart \& Bruyer, 1991), 

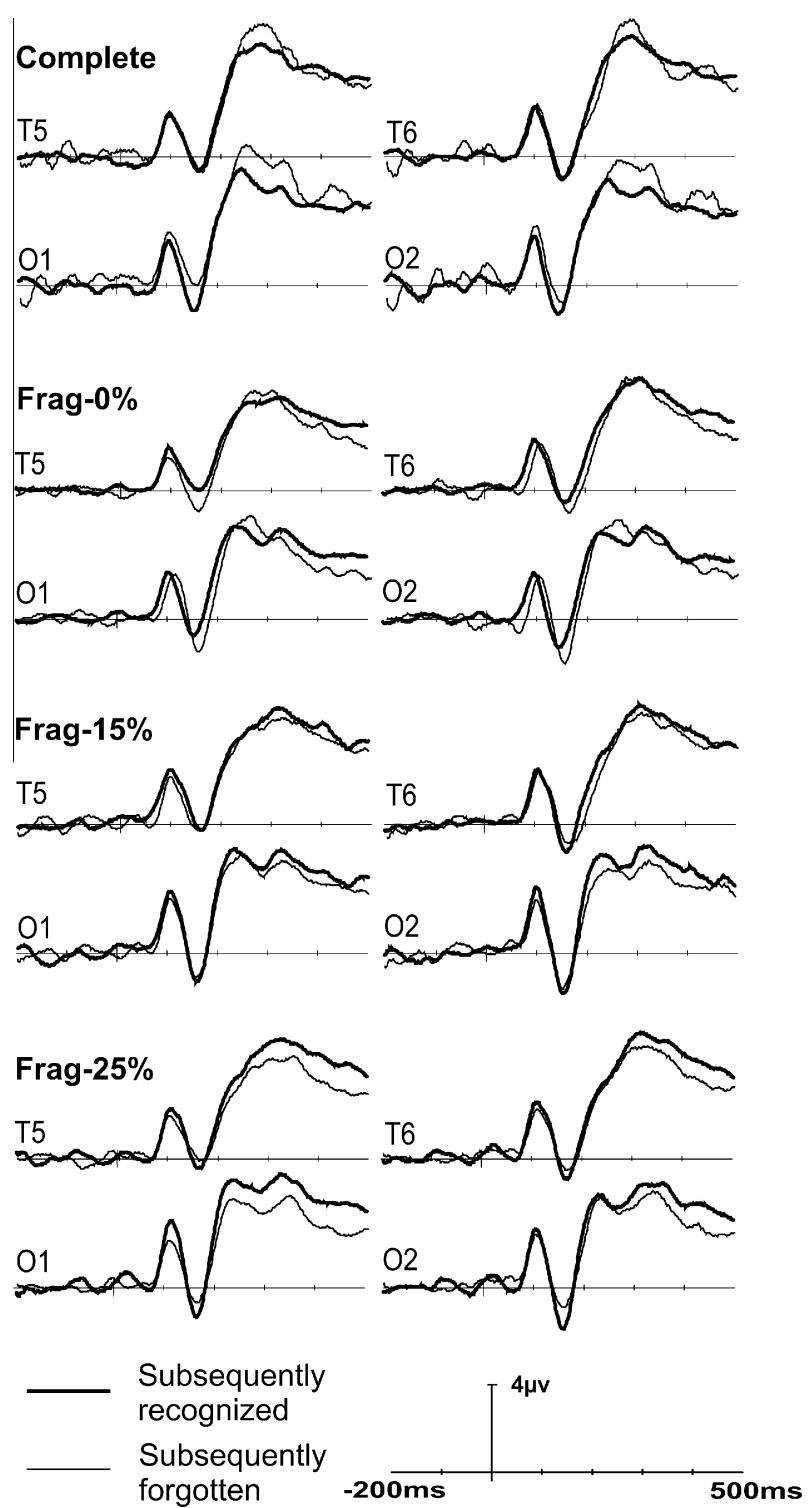

Fig. 5. Grand averaged ERPs $(n=24)$ evoked by the subsequent recognition as a function of fragmentation.

after showing that participants were not significantly impaired by slight fragmentation while completing a sample-matching task. In their study, slight fragmentation was achieved with gaps of lengths equal to $40 \%$ of the fragments' lengths. Gaps started to affect the matching at $100 \%$ of the fragments' lengths. In the current experiment, the length of gaps in Frag-0\% figures was 70\%, and thus likely not high enough to hinder performance. Moreover, one must consider the fact that symmetry was not changed by the fragmentation in the Frag-0\% figures. Fragments were indeed all placed in mirror image fashion. Removing fragments, such as in the Frag15\% and Frag-25\% conditions, disrupted this symmetry and thus explains why reaction times were increased in these conditions.

\subsection{The effect of fragmentation on memory recognition}

The way the occipito-temporal $N_{\text {frag }}$ effect was changed by subsequent recognition has several implications on the interpretation of the processes underlying this ERP component. These processes are unlikely to reflect some sort of closure or filling-in process (Doniger et al., 2000) because in such a case it would have been larger for stimuli that were subsequently recognized. Stimuli that are perceptually closed are more physically similar to complete figure (Snodgrass \& Hirshman, 1994) and as a consequence they should be retrieved from memory more easily when presented in their complete version during the recognition phase. An alternative interpretation is that the occipito-temporal $N_{\text {frag }}$ effect reflects an effort to manage fragmentation and the requirement for additional brain resources. These resources would sometime be sufficient to manage fragmentation, but they could sometime fail and lower the chance of later recognition, thus explaining why the $N_{\text {frag }}$ effect was limited to subsequently forgotten stimuli. According to Schendan and Kutas (2002, see also Pietrowsky et al., 1996; Stuss et al., 1992) this effort could be the search for hypotheses regarding the global structure of the figures. Such search necessarily comes with longer reaction times, which were not observed for the Frag-0\% figures, although it elicited an occipito-temporal $N_{\text {frag. }}$. A conclusion solely based on the search for hypotheses assumption might thus be premature. The occipito-temporal $N_{\text {frag }}$ may alternatively reflect modifications at the phenomenological level (Brodeur et al., 2008a). These modifications would be necessary to elaborate the percept as it is experienced and would warrant its representation being kept in memory. All fragmented figures would activate these modifications but only the Frag-15\% and Frag-25\% figures would rely on them to be discriminated as a function of their symmetry. In contrast, because the global shape of the Frag-0\% figures was not altered, symmetry could be easily and quickly determined without having to rely on the final phenomenological output. This explanation is consistent with the way the $N_{\text {frag }}$ and the occipito-temporal $N_{\text {frag }}$ were modulated across the conditions but it is undermined by the absence of an occipito-temporal $N_{\text {frag }}$ effect for the figures that were subsequently recognized.

Shedding lights on the absence of an occipito-temporal $N_{\text {frag }}$ effect to subsequently recognized figures is the key to a comprehension of the occipito-temporal $N_{\text {frag }}$ effect. One likely reason for this result may relate to the attribution of semantic value to many abstract figures. As mentioned in the methods, standardization of figures showed that each stimulus was rated meaningful only by an average of $14 \%$ students. However, we can assume that in the context of a difficult task requiring subjects to memorize stimuli, this average would be substantially higher. The encoding of figures beneficiating from semantic association will likely require less fragmentation processing and will also likely be better recognized. On the other hand, figures subsequently forgotten may include those for which subjects found no semantic value and which encoding largely depends on the processing of fragmentation. Such encoding strategy is not as efficient as an encoding strategy based on semantic association (Voss, Schendan, \& Paller, 2010). Accordingly, it will systematically lead to a higher forgetting rate of stimuli in the recognition task. The occipito-temporal $N_{\text {frag }}$ effect may thus essentially be evoked by meaningless stimuli (i.e., forgotten stimuli) and be prevented by semantic association (i.e., subsequently recognized stimuli).

It is noteworthy that the modulation of the occipito-temporal $N_{\text {frag }}$ effect as a function of subsequent recognition was partly driven by complete figures which evoked more positive ERPs when subsequently forgotten than recognized. It has long been known that ERPs can be modulated as a function of subsequent recognition (Sanquist, Rohrbaugh, Syndulko, \& Lindsley, 1980). This effect, called Dm (i.e., difference due to memory), is generally reflected by more positive voltage over the frontal area for stimuli that are subsequently recognized (Paller, Kutas, \& Mayes, 1987), a finding contrary to the modulation found with the present complete figures. This discrepancy can be explained by the abstractness of the present stimuli. The Dm effect is generally obtained with semantic stimuli, such as words and pictures of objects (Duarte, Ranganath, Winward, Hayward, \& Knight, 2004; Friedman \& Johnson, 2000; 
Paller, McCarthy, \& Wood, 1988). When using meaningless stimuli, the Dm effect either disappears or takes an opposite direction. For instance, Van Petten and Senkfor (1996) reported a Dm with words but not with abstract figures. Otten and colleagues (2007) described the Dm to nonwords as a negative-going waveform modulated in opposite direction to what was observed with words.

Fig. 5 shows a significant Dm with the complete figures, no Dm with the Frag-0\% and Frag-15\% figures, and a significant inverted Dm with the Frag-25\% figures. These modulations must however be interpreted with caution, because they result not only from the influence of the subsequent memory effect but also from the fragmentation processing activated by forgotten figures at various degrees across conditions. The $\mathrm{Dm}$ is a positive-going effect which disappears once combined with the negative-going occipitotemporal $N_{\text {frag }}$ activated by the Frag- $0 \%$ and Frag- $15 \%$ figures. It is even overruled by the occipito-temporal $N_{\text {frag }}$ with the Frag- $25 \%$ figures which require more fragmentation processing than the other figures. Accordingly, the ERP effects to subsequent recognition are driven both by the subsequent memory effect and fragmentation processing.

In conclusion, stimuli evoking occipito-temporal $N_{\text {frag }}$ will likely be more forgotten than stimuli requiring no such activity. In the present study, the occipito-temporal $N_{\text {frag }}$ may reflect the processing of fragmentation that could not be prevented by attributing meaning to the stimuli. ERPs to fragmentation are thus predictive of memory recognition. It is, however, important to recall that the use of abstract figures and the variation of fragmentation within the limits of recoverability had important influences on the modulation of the occipito-temporal $N_{\text {frag. }}$. Further testing may thus be necessary before concluding that such modulation would be observed with fragmented pictures of known objects presented according to the ascending method of limits.

\section{Acknowledgments}

This research was supported by the Natural Sciences and Engineering Research Council of Canada (Grant \#238617). We are grateful to Thomas Howells for manuscript editing.

\section{References}

Boucart, M., \& Bruyer, R. (1991). Influence of physical and semantic information in a categorisation task of fragmented forms. Perception, 20(3), 403-414.

Boucart, M., Delord, S., \& Giersch, A. (1994). The computation of contour information in complex objects. Perception, 23(4), 399-409.

Boucart, M., \& Humphreys, G. W. (1994). Attention to orientation, size, luminance, and color: Attentional failure within the form domain. Journal of Experimental Psychology: Human, Perception and Performance, 20(1), 61-80.

Brodeur, M., Bacon, B. A., Renoult, L., Prevost, M., Lepage, M., \& Debruille, J. B. (2008a). On the functional significance of the P1 and N1 effects to illusory figures in the notch mode of presentation. PLOS ONE, 3(10), e3505.

Brodeur, M., Pelletier, M. \& Lepage, M. (2008b). Seeing is remembering: Do deficits in closure affect visual memory recognition in schizophrenia? Cognitive Neuropsychiatry, 13(5), 385-405.

Doniger, G. M., Foxe, J. J., Murray, M. M., Higgins, B. A., Snodgrass, J. G., Schroeder, C. E., et al. (2000). Activation timecourse of ventral visual stream object- recognition areas: High density electrical mapping of perceptual closure processes. Journal of Cognitive Neuroscience, 12(4), 615-621.

Duarte, A., Ranganath, C., Winward, L., Hayward, D., \& Knight, R. T. (2004). Dissociable neural correlates for familiarity and recollection during the encoding and retrieval of pictures. Cognitive Brain Research, 18(3), 255-272.

Foley, M. A., Foley, H. J., Durso, F. T., \& Smith, N. K. (1997). Investigations of closure processes: What source-monitoring judgments suggest about what is "closing". Memory and Cognition, 25(2), 140-155.

Friedman, D., \& Johnson, R. Jr., (2000). Event-related potential (ERP) studies of memory encoding and retrieval: A selective review. Microscopy Research and Technique, 51(1), 6-28.

Hess, R., \& Field, D. (1999). Integration of contours: New insights. Trends in Cognitive Sciences, 3(12), 480-486.

Kinjo, H., \& Snodgrass, J. G. (2000). Does the generation effect occur for pictures? American Journal of Psychology(113), 95-121.

Mathes, B., Trenner, D., \& Fahle, M. (2006). The electrophysiological correlate of contour integration is modulated by task demands. Brain Research, 1114(1), 98-112.

Otten, L. J., Sveen, J., \& Quayle, A. H. (2007). Distinct patterns of neural activity during memory formation of nonwords versus words. Journal of Cognitive Neuroscience, 19(11), 1776-1789.

Paller, K. A., Kutas, M., \& Mayes, A. R. (1987). Neural correlates of encoding in an incidental learning paradigm. Electroencephalography and Clinica Neurophysiology, 67(4), 360-371.

Paller, K. A., McCarthy, G., \& Wood, C. C. (1988). ERPs predictive of subsequent recall and recognition performance. Biological Psychology, 26(1-3), 269-276.

Pietrowsky, R., Kuhmann, W., Krug, R., Molle, M., Fehm, H. L., \& Born, J. (1996) Event-related brain potentials during identification of tachistoscopically presented pictures. Brain and Cognition, 32(3), 416-428.

Sanquist, T. F., Rohrbaugh, J. W., Syndulko, K., \& Lindsley, D. B. (1980). Electrocortical signs of levels of processing: Perceptual analysis and recognition memory. Psychophysiology, 17(6), 568-576.

Schendan, H. E., \& Kutas, M. (2002). Neurophysiological evidence for two processing times for visual object identification. Neuropsychologia, 40(7), 931-945.

Sehatpour, P., Molholm, S., Javitt, D. C., \& Foxe, J. J. (2006). Spatiotemporal dynamics of human object recognition processing: An integrated high-density electrica mapping and functional imaging study of "closure" processes. Neuroimage 29(2), 605-618.

Snodgrass, J. G., \& Feenan, K. (1990). Priming effects in picture fragment completion: Support for the perceptual closure hypothesis. Journal of Experimental Psychology: General, 119(3), 276-296.

Snodgrass, J. G., \& Hirshman, E. (1994). Dissociations among implicit and explicit memory tasks: The role of stimulus similarity. Journal of Experimental Psychology: Learning, Memory and Cognition, 20(1), 150-160.

Snodgrass, J. G., \& Kinjo, H. (1998). On the generality of the perceptual closure effect. Journal of Experimental Psychology: Learning, Memory and Cognition, 24(3), 645-658.

Snodgrass, J. G., Smith, B., Feenan, K., \& Corwin, J. (1987). Fragmenting pictures on the Apple MacIntosh computer for experimental and clinical applications. Behavior Research Methods, Instruments and Computers(19), 270-274.

Stuss, D. T., Picton, T. W., Cerri, A. M., Leech, E. E., \& Stethem, L. L. (1992). Perceptual closure and object identification: electrophysiological responses to incomplete pictures. Brain and Cognition, 19(2), 253-266.

Van Petten, C., \& Senkfor, A. J. (1996). Memory for words and novel visual patterns: repetition, recognition, and encoding effects in the event-related brain potential. Psychophysiology, 33(5), 491-506.

Viggiano, M. P., \& Kutas, M. (1998). The covert interplay between perception and memory: event-related potential evidence. Electroencephalography and Clinical Neurophysiology, 108(5), 435-439.

Viggiano, M. P., \& Kutas, M. (2000). Overt and covert identification of fragmented objects inferred from performance and electrophysiological measures. Journal of Experimental Psychology: General, 129(1), 107-125.

Voss, J. L., Baym, C. L., \& Paller, K. A. (2008). Accurate forced-choice recognition without awareness of memory retrieval. Learning and Memory, 15(6), 454-459.

Voss, J. L., Schendan, H. E., \& Paller, K. A. (2010). Finding meaning in novel geometric shapes influences electrophysiological correlates of repetition and dissociates perceptual and conceptual priming. Neuroimage, 49(3), 2879-2889. 